\title{
Systematic Review: Perioperative Systemic Therapy for Metastatic Renal Cell Carcinoma
}

\author{
Patrick G. Pilié and Eric Jonasch* \\ University of Texas, MD Anderson Cancer Center, Houston, TX, USA
}

\begin{abstract}
.
Background: Approximately $16 \%$ of patients with renal cell carcinomas (RCC) present with stage IV disease at time of diagnosis. Treatment options for metastatic clear cell RCC, the most common histologic subtype, have proliferated over the past decade and include a combination of surgery and systemic therapy. The selection of systemic agent and best timing of systemic therapy in relation to nephrectomy is an area of active research.

Objective: To evaluate the evidence for perioperative systemic therapy, including presurgical and postsurgical, for metastatic RCC.

Methods: A systematic literature search using PubMed and MEDLINE databases was performed in January 2017 for articles related to perioperative systemic therapy in metastatic RCC using key word search terms. The authors screened the search results and identified selected publications by predetermined inclusion criteria and consensus. Expert opinion was obtained to assess for publications missed by search.

Results: Early phase clinical trials of antiangiogenic tyrosine kinase inhibitors prior to cytoreductive nephrectomy in select patients show that these systemic agents are safe and effective in the presurgical setting. There are no randomized data evaluating pre- or post-surgical systemic therapy in metastatic RCC.

Conclusions: Retrospective and early-phase prospective studies on the use and timing of systemic therapy in relation to cytoreductive nephrectomy in metastatic RCC show that standard of care antiangiogenic agents are safe and effective in the perioperative setting, though randomized data are still lacking. Pre-surgical immune checkpoint therapy for metastatic RCC has strong biologic rationale and holds promise. Sequential tumor sampling in neoadjuvant and presurgical trials is necessary to determine biomarkers of response and resistance.
\end{abstract}

Keywords: Renal cell carcinoma, RCC, presurgical therapy, nephrectomy, toxicity, immune therapy

\section{INTRODUCTION}

Cancers of the kidney and renal pelvis account for approximately $3.5 \%$ of all new cancer cases in the United States and are responsible for 3.9 deaths per 100,000 individuals per year. Most arise in the

\footnotetext{
*Correspondence to: Eric Jonasch, Department of Genitourinary Medical Oncology, Division of Cancer Medicine, University of Texas, MD Anderson Cancer Center, Houston, TX, USA. E-mail: EJonasch@mdanderson.org.
}

kidney, and are commonly described as renal cell carcinoma (RCC). The majority (65\%) of RCCs present as localized (stage I) disease, and approximately $16 \%$ are diagnosed as de novo metastatic disease (stage IV). The prognosis of RCC is highly dependent on the stage at diagnosis with $92 \%$ of individuals with localized disease alive at 5 years, while only $11.7 \%$ of individuals with metastatic disease survive 5 years $[1,2]$. In addition, the histological subtype of RCC impacts prognosis and treatment with clear cell RCC 
(ccRCC) being the most common subtype, accounting for over $70 \%$ of all RCC [3]. The most common genetic event in ccRCC carcinogenesis is loss of the von Hippel Lindau (VHL) gene, a key tumor suppressor on chromosome $3 \mathrm{p} 25$ with one of its main functions being to downregulate hypoxia inducible factor 1 alpha (HIF1 $\alpha$ ) and 2 alpha (HIF $2 \alpha)$ via VHL ubiquitinating HIF leading to proteasomal degradation [4]. HIF $1 \alpha$ and HIF2 $\alpha$ are crucial pro-angiogenic transcription factors with multiple downstream angiogenic and metabolic targets including vascular endothelial growth factor (VEGF), platelet derived growth factor (PDGF), fibroblast growth factor (FGF), and GLUT1, factors which aid in renal cell carcinogenesis initiation and progression $[4,5]$. Thus the standard of care treatment options for firstline therapy in metastatic ccRCC target dysregulated angiogenesis and metabolism, though currently we lack synthetic lethal systemic treatment strategies for RCC [3].

As is the case with many cancer subtypes, the treatment of localized RCC is primarily surgical; and, there are currently no standard perioperative neoadjuvant or adjuvant systemic treatment approaches for localized RCC [3, 6]. However, unlike most other cancer subtypes, surgery in the form of radical or partial nephrectomy is also part of a multidisciplinary treatment approach for metastatic RCC in select patients with good performance status. Nephrectomy has demonstrated a survival benefit in prospective trials and retrospective reviews of patients with metastatic RCC in both the earlier immune therapy era, as well as the current tyrosine kinase inhibitor (TKI) era [7-9]. However, studies have consistently shown that nephrectomy provides little to no benefit for individuals with poor prognosis and/or poor performance status [7-10]. In the metastatic setting, systemic therapy in the form of an anti-angiogenic tyrosine kinase inhibitor is typically initiated following nephrectomy with the choice and timing of systemic therapy in relation to surgery being somewhat case dependent, and remains an area of active research [11]. Retrospective studies have shown benefit to nephrectomy and targeted therapy versus targeted therapy alone [10]. While the safety and efficacy of presurgical targeted anti-angiogenic therapy has been evaluated in retrospective and Phase I/II studies, we currently lack any Phase III randomized trial data to support or refute its use as a standard of care. Large randomized trials comparing cytoreductive nephrectomy with tyrosine kinase inhibitors (both presurgical and postsurgical) versus tyrosine kinase inhibitors alone are ongoing [NCT00930033 (CARMENA)] or have been closed prematurely [NCT01099423 (SURTIME)], and current guidelines recommend cytoreductive nephrectomy for patients with good performance status and low burden of metastatic disease $[3,12]$. In this systematic review, we will evaluate and summarize published literature and data on the use of presurgical and postsurgical systemic therapy in the setting of metastatic RCC.

\section{METHODS}

We performed a search of Medline and PubMed from inception through 01/2017 under the direction of a medical librarian for prospective clinical trials as well as retrospective studies and retrospective reviews related to perioperative systemic therapy in metastatic RCC. The search was restricted to Englishlanguage articles only. Key search terms included kidney cancer, renal cell carcinoma, metastatic, presurgical, adjuvant, neoadjuvant, perioperative, systemic therapy, immune therapy, tyrosine kinase inhibitors, nephrectomy, metastasectomy, safety, efficacy, randomized control trial. Input from clinical experts in RCC was also obtained to assess for any publications or studies missed by the search.

\section{RESULTS}

Initial search yielded over approximately 740 articles for evaluation. We then filtered for randomized control trials, retrospective studies, systematic reviews/meta-analyses specifically related to presurgical and/or perioperative therapy in metastatic renal cell carcinoma and also included input from experts in the field [13-25].

\section{PRESURGICAL THERAPY IN METASTATIC RCC}

Presurgical therapy in the metastatic RCC setting can serve to identify those patients who are not actively progressing or decompensating, to better select those individuals for nephrectomy. In addition, a presurgical systemic therapy approach as outlined in Fig. 1 can give insight into the biology of a patient's tumor that can aid in future systemic treatment planning and allow for earlier systemic therapy than waiting until post-surgery. Molecular profiling of tumor tissue obtained prior to initiation of presurgical systemic therapy and then again at 


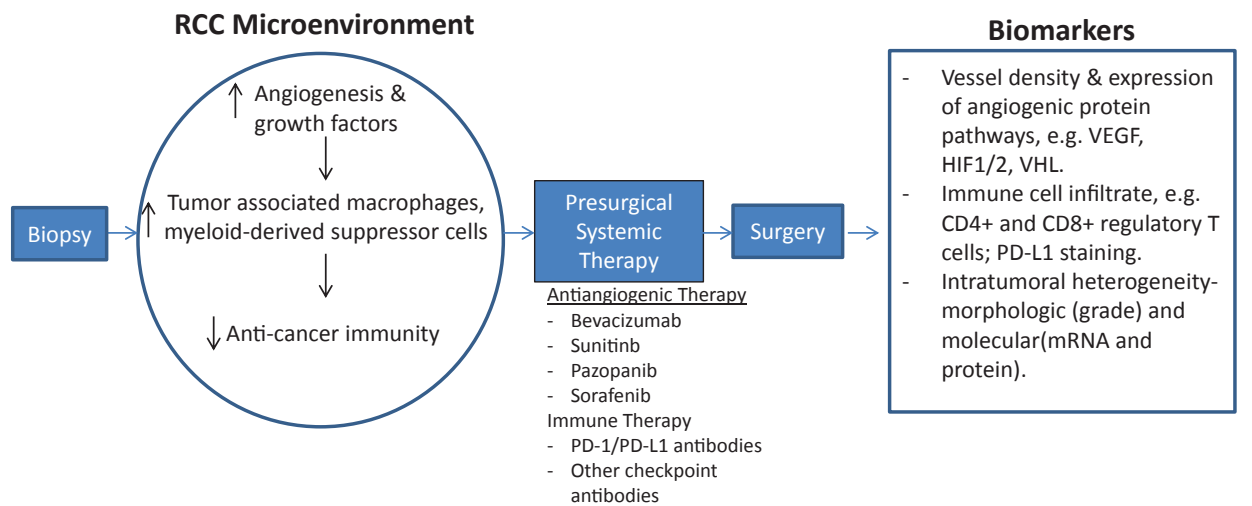

Fig. 1. Schematic of early-phase clinical trial for presurgical systemic antiangiogenic therapy in metastatic RCC. Sequential tissue acquisition pre and post systemic therapy allows for identification of biologic drivers of cancer progression as well as predictive and prognostic biomarker development.

time of surgery can help identify predictive biomarkers of sensitivity and resistance, as well as detect molecular changes associated with clinical outcomes $[23,24]$. However, a pre-surgical treatment strategy necessitates interruption of systemic therapy in the perioperative period, which may increase likelihood of tumor progression. In addition, prior studies have shown that pre-surgical antiangiogenic therapy may lead to an increase in post-surgical complications due delayed wound healing, though other studies show no evidence of excessive post-surgical morbidity or mortality [16, 26-28].

An early pilot study of pre-surgical interferonalpha based immunotherapy in patients with metastatic ccRCC and a primary tumor in situ showed that those patients who did not progress on immunotherapy and were able to undergo nephrectomy had better overall survival 11.5 months versus 3 months) than those who progressed while on immunotherapy. In addition, there was no increased surgical morbidity in patients who received presurgical immunotherapy. This pilot study highlights how pre-surgical therapy can better select patients for nephrectomy and that individuals with progressive disease are considerably less likely to benefit from nephrectomy [21].

Following the introduction of anti-angiogenic and targeted therapy agents for RCC, a phase II study of presurgical bevacizumab in treatment-naïve patients with metastatic ccRCC was performed to test if 1) presurgical bevacizumab was safe and if 2) presurgical anti-angiogenic therapy could select patients who would benefit from cytoreductive nephrectomy [15]. This single-arm pilot study had primary endpoints of time to disease progression and safety. The study included 23 patients who received bevacizumab plus erlotinib and 27 patients who received bevacizumab alone, with $82 \%$ of the study population having intermediate-risk features by Memorial Sloan Kettering Cancer Center (MSKCC) criteria. Median progression free survival (PFS) was 11 months, which met predetermined outcome measures for improvement in PFS attributable to bevacizumab. The 50 patients included had an overall response rate (ORR) of $12 \%$ and median overall survival (OS) of 25.4 months. Ultimately, $84 \%$ of the study population underwent nephrectomy with no intraoperative complications attributable to the study drug(s); however, $20.9 \%$ of those individuals who underwent nephrectomy had delayed wound healing at post-operative week 4. Thus this study showed that outcomes of patients treated pre-surgically with bevacizumab were similar to outcomes in groups treated postsurgically, but also suggests that at least this particular agent contributes to delayed wound healing when compared to retrospective historical controls. Since single-agent bevacizumab is not considered a first line option for metastatic ccRCC and is rarely used even in second line setting in current clinical practice, this study provides general guidance on the use of antiangiogenic therapy in the presurgical setting but does not directly reflect current treatment of RCC.

A prospective pilot study of sorafenib, a multitargeted TKI, was performed in a preoperative population with stage II or higher renal masses, including 17 patients with localized disease and 13 with metastatic disease, which also included nonclear cell histologies [25]. 28/30 of these patients were evaluable for response to sorafenib prior to surgery with $2 / 28$ having a partial response (PR) and 
26/28 with stable disease (SD). Interestingly thirty patients were able to proceed with nephrectomy, and there were no surgical complications related to sorafenib administration.

Sunitinib is a multiple receptor TKI that is widely used in the setting of metastatic RCC. In a randomized phase III trial of sunitinib versus interferon-alfa for metastatic RCC, patients had a median OS of greater than 2 years in the sunitinib group with a median PFS of 11 months [29]. Two prospective single-arm phase II studies of presurgical sunitinib prior to planned nephrectomy have been published with a variable number of cycles given prior to surgery $[16,30]$. These studies of pre-surgical sunitinib included a combined total of 66 patients with metastatic ccRCC that were treatment naive, with $32 \%$ having MSKCC poor risk disease. The majority of the cohort (73\%) achieved clinical benefit. The median PFS for the intermediate-risk patients was 8 months in the combined cohort, with the poorrisk patients having a PFS of 6.0 months. The OS for the combined cohort was 15.2 months, with the intermediate-risk group having a significantly longer OS than the poor-risk group (26.0 months vs 9.0 months) which is consistent with prior studies with other treatment modalities. Seventy-one percent of the cohort went on to nephrectomy; and, patients in these studies were off systemic therapy perioperatively for approximately 29 days, with a significant minority of assessable patients $(36 \%)$ progressing during or shortly after this required treatment break. In addition $13 \%$ of individuals undergoing nephrectomy had complication of delayed wound healing. Not surprisingly, progression in the primary tumor in situ or in metastatic sites at the time of planned surgery portended for shorter OS.

A recent single-arm, phase II study of pazopaniba tyrosine kinase inhibitor which is a standard of care option in first line treatment of metastatic RCC- in the presurgical setting prior to cytoreductive nephrectomy has shown a favorable safety and efficacy profile [20]. In this study, 104 patients with metastatic ccRCC that were treatment naïve received pazopanib $800 \mathrm{mg}$ daily prior to undergoing cytoreductive nephrectomy $(\mathrm{CN})$, with the study's primary end point being clinical or radiographic response prior to surgery. One hundred patients were included in the final analysis with $84 \%$ of the study population achieving a clinical benefit defined as tumor size reduction or stable disease prior to nephrectomy with a $14.4 \%$ median reduction in the size of the primary kidney tumor. The majority of the study population
(71\%) achieved only stable disease, thus pazopanib did not facilitate for better surgical outcomes in this patient population as a whole. In addition patients with poor risk disease as defined by MSKCC criteria did not benefit from nephrectomy and had very poor outcomes with an OS of 5.7 months. Ultimately, $63 \%$ of the study population underwent nephrectomy with a surgical complication rate of $22 \%$, with the most common complications being bleeding and delayed wound healing. The PFS and OS for the study population were 7.1 months and 22.7 months, respectively, which are similar to studies of pazopanib in the metastatic ccRCC setting following nephrectomy [31, 32].

Each of these presurgical studies not only provided clinical information on patient outcomes in the metastatic ccRCC setting (Table 1), but also allowed for tissue based molecular analyses to elucidate underlying mechanisms of drug sensitivity and resistance, as well as providing a more complete picture of the biology driving tumor growth and progression. A retrospective analysis of RNA microarray and reverse phase protein array profiling of 37 tumors from patients treated in the presurgical bevacizumab plus or minus erlotinib study [15] showed worse OS and PFS in those patients with upregulation of the phosphoinositide 3-kinase (PI3K) pathway and cellcycle related pathways [23].

A subsequent retrospective analysis of tissue obtained from 41 untreated primary RCCs, 42 bevacizumab treated RCCs, and 39 sunitinibtreated RCCs showed that the tumors from patients pretreated with antiangiogenic therapy had increased infiltration of CD4+ and CD8+ T lymphocytes [24]. Those tumors from pretreated patients showed higher infiltration of $\mathrm{CD} 4+\mathrm{FOXP} 3+$ regulatory $\mathrm{T}$ cells and higher expression of programmed death-ligand 1 (PD-L1), and both of these immunosuppressive features were correlated with T-cell infiltration and worse patient survival [24].

The effect of presurgical TKI therapy on ten predetermined biomarkers was assessed in sequential tissue pooled from patients who were pretreated with VEGF TKIs from three phase II studies, including a matched untreated renal biopsy specimen and subsequent treated primary [33]. This study showed TKI treatment resulted in a reduction in vessel density; reduction in phospho-S6K, PD-L1, and FOXP3; and, an increased expression of cytoplasmic FGF-2 and MET receptor in vessels- this highlights the heterogeneous, dynamic molecular changes occurring with TKI therapy [33]. In another tissue-based molecular 
Table 1

General characteristics and outcomes of presurgical systemic therapy studies in mRCC

\begin{tabular}{|c|c|c|c|c|c|c|}
\hline & Study Type & $\begin{array}{c}\text { Presurgical Systemic } \\
\text { Therapy }\end{array}$ & $\begin{array}{l}\text { Patients }{ }^{\mathrm{a}} \\
\text { (n) }\end{array}$ & $\begin{array}{c}\text { Best } \\
\text { Response }\end{array}$ & $\begin{array}{c}\text { Completed } \\
\text { Surgery (\%) }\end{array}$ & $\begin{array}{c}\text { Overall Survival } \\
\text { (months) }\end{array}$ \\
\hline Bex et al. [17] & Phase I/II & Sunitinib & 16 & $\begin{array}{l}\mathrm{CR}^{\mathrm{b}} 0 / 16 \\
\mathrm{PR}^{\mathrm{c}} 2 / 16 \\
\mathrm{SD}^{\mathrm{d}} 9 / 16\end{array}$ & $11(69)$ & 11.5 \\
\hline Jonasch et al. [15] & Phase II & $\begin{array}{l}\text { Bevacizumab } \\
+/- \text { Erlotinib }\end{array}$ & 50 & $\begin{array}{c}\text { CR 1/50 } \\
\text { PR 5/50 } \\
\text { SD 29/50 }\end{array}$ & $42(84)$ & 25.4 \\
\hline Cowey et al. [25] & Phase I/II & Sorafenib & $\begin{array}{c}30 \\
17 \text { Local } \\
13 \text { Metastatic }\end{array}$ & $\begin{array}{c}\text { CR 0/30 } \\
\text { PR 2/30 } \\
\text { SD 26/30 }\end{array}$ & $30(100)$ & $\mathrm{N} / \mathrm{A}^{\mathrm{e}}$ \\
\hline Powles et al. [16] & Phase II & Sunitinib & 66 & $\begin{array}{c}\text { CR 0/66 } \\
\text { PR 13/66 } \\
\text { SD 35/66 }\end{array}$ & $47(71)$ & 15.2 \\
\hline Powles et al. [20] & Phase II & Pazopanib & 104 & $\begin{array}{l}\text { CR 0/100 } \\
\text { PR 13/100 } \\
\text { SD 71/100 }\end{array}$ & $63(61)$ & 22.7 \\
\hline
\end{tabular}

${ }^{\mathrm{a}}$ studies included patients with metastatic disease only unless otherwise specified; ${ }^{\mathrm{b}} \mathrm{CR}=$ complete response; ${ }^{\mathrm{c}} \mathrm{PR}=$ partial response;

${ }^{\mathrm{d}} \mathrm{SD}=$ stable disease $;{ }^{\mathrm{e}} \mathrm{N} / \mathrm{A}=$ not available.

profiling study of sequential tumor samples from primary tumors obtained from patients with metastatic ccRCC pretreated with sunitinib versus untreated control samples, the investigators assessed for intratumoral heterogeneity as defined by morphologic and molecular (including DNA, mRNA, and protein) variance within a single tumor [22]. This study found that morphologic intratumoral grade heterogeneity was present to a higher degree in sunitinib-treated versus untreated samples and that driver-mutation gene signatures and protein expression were increasingly variable in treated samples.

The phase II trial of pazopanib in the presurgical setting in metastatic RCC assessed antibody-based biomarkers of PD-L1, C-MET, HIF1 $\alpha$, VEGFR2, and VHL in sequential tissue specimens from pre and post-pazopanib treatment [20]. They found in comparison of pre and post pazopanib specimens a statistically significant decrease in CD8 expression and reduction in expression of VHL and C-MET with increased PD-L1 expression. No biomarkers in this study were associated with response to therapy.

Thus these pilot and early-phase studies indicate that the approach of pre-surgical targeted therapy is generally safe, can provide disease control albeit predominantly in intermediate-risk cases, and can select patients with poor-risk or actively progressing disease that will not benefit from nephrectomy. In addition molecular studies on tissue from patients that receive presurgical systemic therapy can give insight into underlying biology, resistance mechanisms, as well as predictive and prognostic biomarkers.
Immune checkpoint therapy is currently standard of care in the second line setting for metastatic ccRCC [3] following the phase III study showing nivolumab, an anti-programmed cell death 1 (PD-1) receptor antibody, had an OS benefit compared to everolimus in metastatic ccRCC in second line setting [34]. While prior combinations of antiangiogenic agents and cytokine-based therapies did not show benefit and/or were too toxic, the relatively favorable side effects profile of modern immune checkpoint therapies have led to multiple ongoing trials involving the combination of these immune directed agents with anti-angiogenics [35]. Preclinical studies have shown a signal for increased efficacy of modern immunotherapy in the neoadjuvant setting compared to adjuvant setting due to increased, sustained activation of $\mathrm{T}$ cell antitumor activity with an effector/memory phenotype [36]. The mechanisms by which an intact tumor may serve to prime an antitumor immune response in the setting of neoadjuvant or presurgical therapy are numerous, but a general schematic is that the generation of neoantigens from dying tumor cells can lead to an quantitative expansion of targeted tumor-specific $\mathrm{T}$ cells as well as a sustained qualitative improvement in immune memory even after the tumor is resected. In this setting, the intact tumor is necessary to expand the anti-tumor T cell army, as well as to train these T cells to systemically surveil the body for tumor cells even after the primary tumor or metastasis is removed [36]. Thus, early phase clinical studies are underway to evaluate the safety and efficacy of anti- PD-1 receptor antibody in the presurgical setting both as single-agent ther- 
apy [37] and in combination with anti-VEGF therapy or with ipilimumab, an anti-CTLA4 antibody [38]. A phase I study of nivolumab in the perioperative setting for patients with metastatic RCC for which nephrectomy is planned is ongoing (ADAPTer), with plans to recruit nineteen patients and requires sequential tissue specimens pre- and post-nivolumab therapy [37]. In addition, studies are ongoing to combine radiation with immune therapy in presurgical and metastatic settings with the idea that radiation can further drive neoantigen production and take advantage of potential abscopal effects of local radiation to lead to immune-mediated regression of distant disease $[39,40]$. With a strong preclinical rationale combined with the potential for long-term sustained responses following surgery and predictive immunerelated biomarkers, presurgical immunotherapy both alone and in combination with other therapies in metastatic RCC is very promising.

\section{POSTSURGICAL THERAPY IN METASTATIC RCC}

The current guidelines for metastatic RCC recommend cytoreductive nephrectomy in patients with good performance status and low-volume metastatic disease, which is based on the prospectively confirmed survival benefit of nephrectomy in the era of systemic therapy with interferon alfa [7]. Multiple trials of first-line and subsequent line targeted inhibitors have been performed for metastatic clear cell and non-clear cell histologies, with a significant number of patients in those studies having undergone prior cytoreductive nephrectomy at time of initiation of systemic therapy [29, 31, 41-44]. However, there are no randomized trials completed and reported to date that specifically address perioperative or postsurgical systemic therapy in the setting of nephrectomy versus systemic therapy alone. Multiple randomized control trials of TKIs, including sunitinib and pazopanib, alone versus TKI following nephrectomy are ongoing (CARMENA and TARIBO) [45, 46]. In addition, multiple prospective and retrospective studies suggest that patients with isolated surgically resectable metastatic disease obtain clinical benefit from metastasectomy, even in the era of targeted therapy, though randomized data are lacking [19, 47-49]. Thus in current practice, almost all patients seeking treatment for metastatic RCC will receive systemic therapy at some point in their treatment course, and those with good performance status and who are amenable to surgery would be advised to undergo upfront nephrectomy, and possibly metastasectomy in appropriately selected cases.

\section{CONCLUSIONS}

Metastatic RCC requires a multidisciplinary, team-based treatment approach that incorporates surgical resection and systemic therapy. Cytoreductive nephrectomy in patients with metastatic RCC has been shown to prolong survival in selected patients with good prognosis and performance status; however, large randomized control trial data are still lacking in this space. This review highlights important retrospective and early-phase prospective studies on the use and timing of systemic therapy, including targeted anti-angiogenic agents and immune therapy, prior to cytoreductive nephrectomy. Published data thus far support the efficacy and safety of a presurgical systemic therapy approach using standard of care TKIs including sunitinib or pazopanib, though randomized data are still lacking. Given the rapid proliferation of effective immune checkpoint therapy options for metastatic RCC, the community's increasing familiarity with their use and management of their side effects in the second-line setting, and the strong biologic rationale, it is likely that immune checkpoint therapy will move to the front-line and presurgical settings for metastatic RCC in the near future. The molecular alterations and potential biomarkers for selecting those patients who would benefit most from nephrectomy and specific systemic therapies are evolving but still not well understood, which highlights the necessity and benefit to sequential tumor sampling in neoadjuvant and presurgical clinical trials. Data from current ongoing perioperative trials with TKIs together with immune checkpoint blocking agents will better inform these questions of best choice and timing of systemic treatment in metastatic RCC.

\section{REFERENCES}

[1] Cancer Statistics Review, 1975-2014 - SEER Statistics [Internet][cited 2017 Apr 26] Available from: https://seer. cancer.gov/csr/1975_2014/

[2] Noone A-M, Cronin KA, Altekruse SF, et al. Cancer incidence and survival trends by subtype using data from the surveillance epidemiology and end results program, 19922013. Cancer Epidemiol Biomark Prev Publ Am Assoc Cancer Res Cosponsored Am Soc Prev Oncol 2017;26: 632-41. 
[3] Motzer RJ, Jonasch E, Agarwal N, et al. Kidney cancer, version 2.2017, NCCN clinical practice guidelines in oncology. J Natl Compr Cancer Netw JNCCN 2017;15: 804-34.

[4] Gossage L, Eisen T, Maher ER. VHL, the story of a tumour suppressor gene. Nat Rev Cancer 2015;15:55-64.

[5] Maxwell PH, Wiesener MS, Chang GW, et al. The tumour suppressor protein VHL targets hypoxia-inducible factors for oxygen-dependent proteolysis. Nature 1999;399: 271-5.

[6] Jonasch E. Incorporating new systemic therapies in kidney cancer treatment. J Natl Compr Cancer Netw JNCCN 2017; 15:703-5.

[7] Flanigan RC, Mickisch G, Sylvester R, et al. Cytoreductive nephrectomy in patients with metastatic renal cancer: A combined analysis. J Urol 2004;171:1071-6.

[8] Mickisch GH, Garin A, van Poppel H, et al. Radical nephrectomy plus interferon-alfa-based immunotherapy compared with interferon alfa alone in metastatic renal-cell carcinoma: A randomised trial. Lancet Lond Engl 2001;358: 966-70.

[9] Choueiri TK, Xie W, Kollmannsberger C, et al. The impact of cytoreductive nephrectomy on survival of patients with metastatic renal cell carcinoma receiving vascular endothelial growth factor targeted therapy. J Urol 2011;185: 60-6.

[10] Heng DYC, Wells JC, Rini BI, et al. Cytoreductive nephrectomy in patients with synchronous metastases from renal cell carcinoma: Results from the International Metastatic Renal Cell Carcinoma Database Consortium. Eur Urol 2014;66:704-10.

[11] Ljungberg B, Bensalah K, Canfield S, et al. EAU guidelines on renal cell carcinoma: 2014 update. Eur Urol 2015;67:913-24.

[12] Culp SH. Cytoreductive nephrectomy and its role in the present-day period of targeted therapy. Ther Adv Urol 2015;7:275-85.

[13] Chapin BF, Delacroix SE, Culp SH, et al. Safety of presurgical targeted therapy in the setting of metastatic renal cell carcinoma. Eur Urol 2011;60:964-71.

[14] Harshman LC, Yu RJ, Allen GI, et al. Surgical outcomes and complications associated with presurgical tyrosine kinase inhibition for advanced renal cell carcinoma (RCC). Urol Oncol 2013;31:379-85.

[15] Jonasch E, Wood CG, Matin SF, et al. Phase II presurgical feasibility study of bevacizumab in untreated patients with metastatic renal cell carcinoma. J Clin Oncol Off J Am Soc Clin Oncol 2009;27:4076-81.

[16] Powles T, Blank C, Chowdhury S, et al. The outcome of patients treated with sunitinib prior to planned nephrectomy in metastatic clear cell renal cancer. Eur Urol 2011;60: 448-54.

[17] Bex A, Blank C, Meinhardt W, et al. A phase II study of presurgical sunitinib in patients with metastatic clear-cell renal carcinoma and the primary tumor in situ. Urology 2011;78:832-7.

[18] Karam JA, Rini BI, Varella L, et al. Metastasectomy after targeted therapy in patients with advanced renal cell carcinoma. J Urol 2011;185:439-44.

[19] Daliani DD, Tannir NM, Papandreou CN, et al. Prospective assessment of systemic therapy followed by surgical removal of metastases in selected patients with renal cell carcinoma. BJU Int 2009; 104:456-60.

[20] Powles T, Sarwar N, Stockdale A, et al. Safety and Efficacy of Pazopanib Therapy Prior to Planned Nephrec- tomy in Metastatic Clear Cell Renal Cancer. JAMA Oncol 2016;2:1303-9.

[21] Bex A, Horenblas S, Meinhardt W, et al. The role of initial immunotherapy as selection for nephrectomy in patients with metastatic renal cell carcinoma and the primary tumor in situ. Eur Urol 2002;42:570-4; discussion 575-6.

[22] Stewart GD, O'Mahony FC, Laird A, et al. Sunitinib Treatment Exacerbates Intratumoral Heterogeneity in Metastatic Renal Cancer. Clin Cancer Res Off J Am Assoc Cancer Res 2015;21:4212-23.

[23] Tsavachidou-Fenner D, Tannir N, Tamboli P, et al. Gene and protein expression markers of response to combined antiangiogenic and epidermal growth factor targeted therapy in renal cell carcinoma. Ann Oncol Off J Eur Soc Med Oncol 2010;21:1599-606.

[24] Liu X-D, Hoang A, Zhou L, et al. Resistance to anti-angiogenic therapy is associated with an immunosuppressive tumor microenvironment in metastatic renal cell carcinoma. Cancer Immunol Res 2015;3:1017-29.

[25] Cowey CL, Amin C, Pruthi RS, et al. Neoadjuvant Clinical Trial With Sorafenib for Patients With Stage II or Higher Renal Cell Carcinoma. J Clin Oncol 2010;28:1502-7.

[26] Powles T, Kayani I, Sharpe K, et al. A prospective evaluation of VEGF-targeted treatment cessation in metastatic clear cell renal cancer. Ann Oncol Off J Eur Soc Med Oncol 2013;24:2098-103.

[27] Margulis V, Matin SF, Tannir N, et al. Surgical morbidity associated with administration of targeted molecular therapies before cytoreductive nephrectomy or resection of locally recurrent renal cell carcinoma. J Urol 2008;180: 94-8.

[28] Wood CG, Margulis V. Neoadjuvant (presurgical) therapy for renal cell carcinoma: A new treatment paradigm for locally advanced and metastatic disease. Cancer 2009;115:2355-60.

[29] Motzer RJ, Hutson TE, Tomczak P, et al. Overall survival and updated results for sunitinib compared with interferon alfa in patients with metastatic renal cell carcinoma. J Clin Oncol Off J Am Soc Clin Oncol 2009;27:3584-90.

[30] Powles T, Kayani I, Blank C, et al. The safety and efficacy of sunitinib before planned nephrectomy in metastatic clear cell renal cancer. Ann Oncol Off J Eur Soc Med Oncol 2011;22:1041-7.

[31] Motzer RJ, Hutson TE, Cella D, et al. Pazopanib versus sunitinib in metastatic renal-cell carcinoma. N Engl J Med 2013;369:722-31.

[32] Motzer RJ, Hutson TE, McCann L, et al. Overall survival in renal-cell carcinoma with pazopanib versus sunitinib. N Engl J Med 2014;370:1769-70.

[33] Sharpe K, Stewart GD, Mackay A, et al. The effect of VEGFtargeted therapy on biomarker expression in sequential tissue from patients with metastatic clear cell renal cancer. Clin Cancer Res Off J Am Assoc Cancer Res 2013;19:692434.

[34] Motzer RJ, Escudier B, McDermott DF, et al. Nivolumab versus Everolimus in Advanced Renal-Cell Carcinoma. N Engl J Med 2015;373:1803-13.

[35] Kuusk T, Albiges L, Escudier B, et al. Antiangiogenic therapy combined with immune checkpoint blockade in renal cancer. Angiogenesis 2017;20:205-15.

[36] Liu J, Blake SJ, Yong MCR, et al. Improved efficacy of neoadjuvant compared to adjuvant immunotherapy to eradicate metastatic disease. Cancer Discov 2016;6:1382-99.

[37] A Study of Anti-PD1 (Nivolumab) Therapy as Pre- and Post-operative Therapy in Metastatic Renal Cell Cancer 
(ADAPTeR) - Full Text View - ClinicalTrials.gov [Internet][cited 2017 Jun 18] Available from: https://clinicaltrials. gov/ct2/show/NCT02446860

[38] Nivolumab vs Nivolumab + Bevacizumab vs Nivolumab + Ipilimumab in Metastatic Renal Cell Carcinoma (mRCC) Full Text View - ClinicalTrials.gov [Internet][cited 2017 Jun 18] Available from: https://clinicaltrials.gov/ct2/show/ NCT02210117

[39] Tang C, Wang X, Soh H, et al. Combining Radiation and Immunotherapy: A New Systemic Therapy for Solid tumors? Cancer Immunol Res 2014;2:831-8.

[40] Kang J, Demaria S, Formenti S. Current clinical trials testing the combination of immunotherapy with radiotherapy. J Immunother Cancer 2016;4:51.

[41] Armstrong AJ, Halabi S, Eisen T, et al. Everolimus versus sunitinib for patients with metastatic non-clear cell renal cell carcinoma (ASPEN): A multicentre, open-label, randomised phase 2 trial. Lancet Oncol 2016;17:378-88.

[42] Choueiri TK, Halabi S, Sanford BL, et al. Cabozantinib versus sunitinib as initial targeted therapy for patients with metastatic renal cell carcinoma of poor or intermediate risk: The alliance A031203 CABOSUN trial. J Clin Oncol Off J Am Soc Clin Oncol 2017;35:591-7.

[43] Hutson TE, Lesovoy V, Al-Shukri S, et al. Axitinib versus sorafenib as first-line therapy in patients with metastatic renal-cell carcinoma: A randomised open-label phase 3 trial. Lancet Oncol 2013;14:1287-94.

[44] Voss MH, Bhatt RS, Plimack ER, et al. The DART study: Results from the dose-escalation and expansion cohorts evaluating the combination of dalantercept plus axitinib in advanced renal cell carcinoma. Clin Cancer Res Off J Am Assoc Cancer Res 2017;14:3557-65.

[45] Clinical Trial to Assess the Importance of Nephrectomy Full Text View - ClinicalTrials.gov [Internet][cited 2017 May 29] Available from: https://clinicaltrials.gov/ct2/show/ NCT00930033

[46] Targeted Therapy With or Without Nephrectomy in Metastatic Renal Cell Carcinoma: Liquid Biopsy for Biomarkers Discovery - Full Text View -ClinicalTrials.gov [Internet][cited 2017 May 29] Available from: https://clini caltrials.gov/show/NCT02535351

[47] Dabestani S, Marconi L, Hofmann F, et al. Local treatments for metastases of renal cell carcinoma: A systematic review. Lancet Oncol 2014;15:e549-61.

[48] Thomas AZ, Adibi M, Borregales LD, et al. Role of metastasectomy in metastatic renal cell carcinoma. Curr Opin Urol 2015;25:381-9.

[49] Kavolius JP, Mastorakos DP, Pavlovich C, et al. Resection of metastatic renal cell carcinoma. J Clin Oncol Off J Am Soc Clin Oncol 1998;16:2261-6. 